\title{
Masers and Galactic structure: Micro-arcsecond astrometry with the VLBA
}

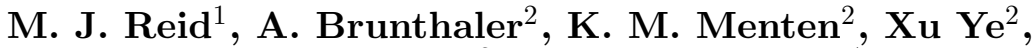 \\ Zheng Xing-Wu $\mathbf{W}^{3}$ and L. Moscadelli ${ }^{4}$ \\ ${ }^{1}$ Harvard-Smithsonian CfA, Cambridge, MA, USA \\ email: reid@cfa.harvard.edu \\ ${ }^{2}$ MPIfR, Bonn, Germany \\ ${ }^{3}$ Nanjing University, Nanjing, China \\ ${ }^{4}$ Arcetri Observatory, Firenze, Italy
}

\begin{abstract}
Astrometric observations with the VLBA with accuracies approaching $\sim 10 \mu$ as are being conducted in order to better understand the Galaxy. The location of Sgr A* on infrared images can be determined with an accuracy of a few mas, using stars with $\mathrm{SiO}$ maser emission as a calibration grid for infrared images. The apparent proper motion of $\mathrm{Sgr} \mathrm{A}^{*}$, which is dominated by the effects of the orbit of the Sun around the Galactic center, has been measured with high accuracy. This measurement strongly constrains $\Theta_{0} / R_{0}$ and offers a dynamical definition of the Galactic plane with $\operatorname{Sgr} \mathrm{A}^{*}$ at its origin. The intrinsic motion of $\operatorname{Sgr} \mathrm{A}^{*}$ is very small and comparable to that expected for a supermassive black hole. When combined with infrared results, this provides overwhelming evidence that $\mathrm{Sgr} \mathrm{A}^{*}$ is a supermassive black hole. Finally, we are engaged in a large project to map the spiral structure and kinematics of the Galaxy. Preliminary trigonometric parallaxes, obtained with the VLBA, to eight massive star forming regions are presented.
\end{abstract}

Keywords. Galactic structure, supermassive black holes, $\mathrm{SiO}$ masers, methanol masers, astrometry, VLBA, parallaxes, proper motions

\section{Where is Sgr A*(IR)?}

Sgr A*, the supermassive black hole that anchors the center of the Galaxy, is a strong radio source but quite dim at infrared wavelengths. This makes it difficult to locate Sgr A* on infrared images: Sgr A*(IR). Also, the central stellar cluster is quite dense, and these stars move considerably from year to year, further complicating locating Sgr A*(IR). In order to transfer the highly accurate radio position of $\operatorname{Sgr} \mathrm{A}^{*}$ to infrared images, one needs a grid of stars near Sgr $\mathrm{A}^{*}$ visible at both radio and infrared wavelengths. Fortunately, this is available as Mira-like variables found in the Galactic center are both strong infrared sources and have circumstellar masers that are strong radio sources.

Menten et al. (1997) provided the first highly accurate position of Sgr A*(IR) relative to stars in the Galactic center. We have been continuing this effort in order to find more $\mathrm{SiO}$ maser stars and to improve upon the accuracy of their position and proper motions. Fig. 1, from Reid et al. (2007), shows a composite spectrum of $\mathrm{SiO} v=1, \mathrm{~J}=1-0$ maser emission from 15 stars within $\approx 45^{\prime \prime}$ of Sgr A*. This spectrum was taken with the National Radio Astronomy Observatory's $\dagger$ VLA on three observing runs between 1998 and 2006.

$\dagger$ The National Radio Astronomy Observatory is operated by Associated Universities, Inc., under a cooperative agreement with the National Science Foundation. 

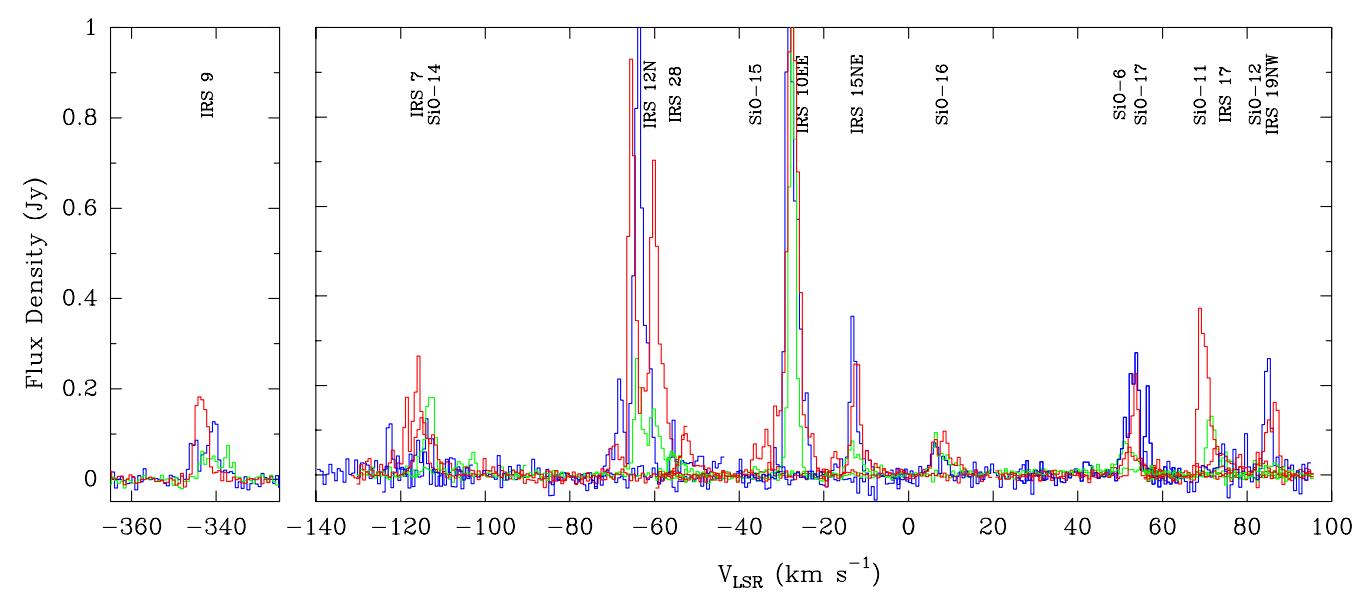

Figure 1. Composite spectrum of stellar $\mathrm{SiO}$ masers detected with the VLA in 1998, 2000, and 2006. Stars are identified at the top. Taken from Reid et al. (2007).

These masers are variable, as expected from observations of Mira-like variables in the solar neighborhood.

We have been measuring the positions of these stars, some for nearly a decade. Not all masers are detected at all epochs, and our position and motion accuracies depend on the strength of the masers and on the time span of observations with detections. A typical position accuracy is $\approx 1$ mas and proper motion accuracy is $\sim 0.3$ mas $y^{-1}$. The locations of the nine maser stars within $20^{\prime \prime}$ of Sgr A* are shown in Fig. 2.

The maser stars provide a critical calibration "grid" for the measurement of stellar orbits in the central milli-pc with infrared techniques. Since the stars move rapidly, continued monitoring is needed to avoid long extrapolations using measured proper motions. Currently, the $\mathrm{SiO}$ spectra are pieced together by splitting the observing time among about ten $3-\mathrm{MHz}$ bands, in order to achieve adequate spectral resolution. The upcoming EVLA capabilities of broad bandwidth and high spectral resolution will greatly enhance future observations.

One star, IRS 9, has a very large 3-D motion of $370 \mathrm{~km} \mathrm{~s}^{-1}$ at a projected distance of $0.33 \mathrm{pc}$ from $\mathrm{Sgr} \mathrm{A}^{*}$. If bound to the central pc, this requires an excess of nearly $10^{6} \mathrm{M}_{\odot}$ above that attributed to Sgr $\mathrm{A}^{*}$ and the observed stellar cluster. Possible explanation for this anomaly include 1) that IRS 9 is not bound to the central parsec, but is on a highly elliptical orbit that takes it out to $\approx 10 \mathrm{pc} ; 2$ ) that a cluster of dark stellar remnants accounts for the missing mass; and/or 3) that the galactocentric distance $R_{0}$ is considerably greater than $8 \mathrm{kpc}$. These and other possibilities are discussed in detail by Reid et al. (2007).

\section{The proper motion of $\operatorname{Sgr} A^{*}$}

We have been measuring the proper motion of the compact radio source Sgr $\mathrm{A}^{*}$ since 1995. In Fig. 3 we show the expected apparent motion for Sgr A*, relative to two background quasars, on a $90 \mathrm{~cm}$ wavelength image of the Galactic center. The apparent motion stems from our moving vantage point near the Sun, which orbits the Galactic center about every 225 My.

Were Sgr A* a stellar mass object, for example a mass transfer binary with a compact object such as a neutron star or black hole, it would be moving at speeds of many thousands of $\mathrm{km} \mathrm{s}^{-1}$, as observed for stars in the deep gravitational potential. However, 


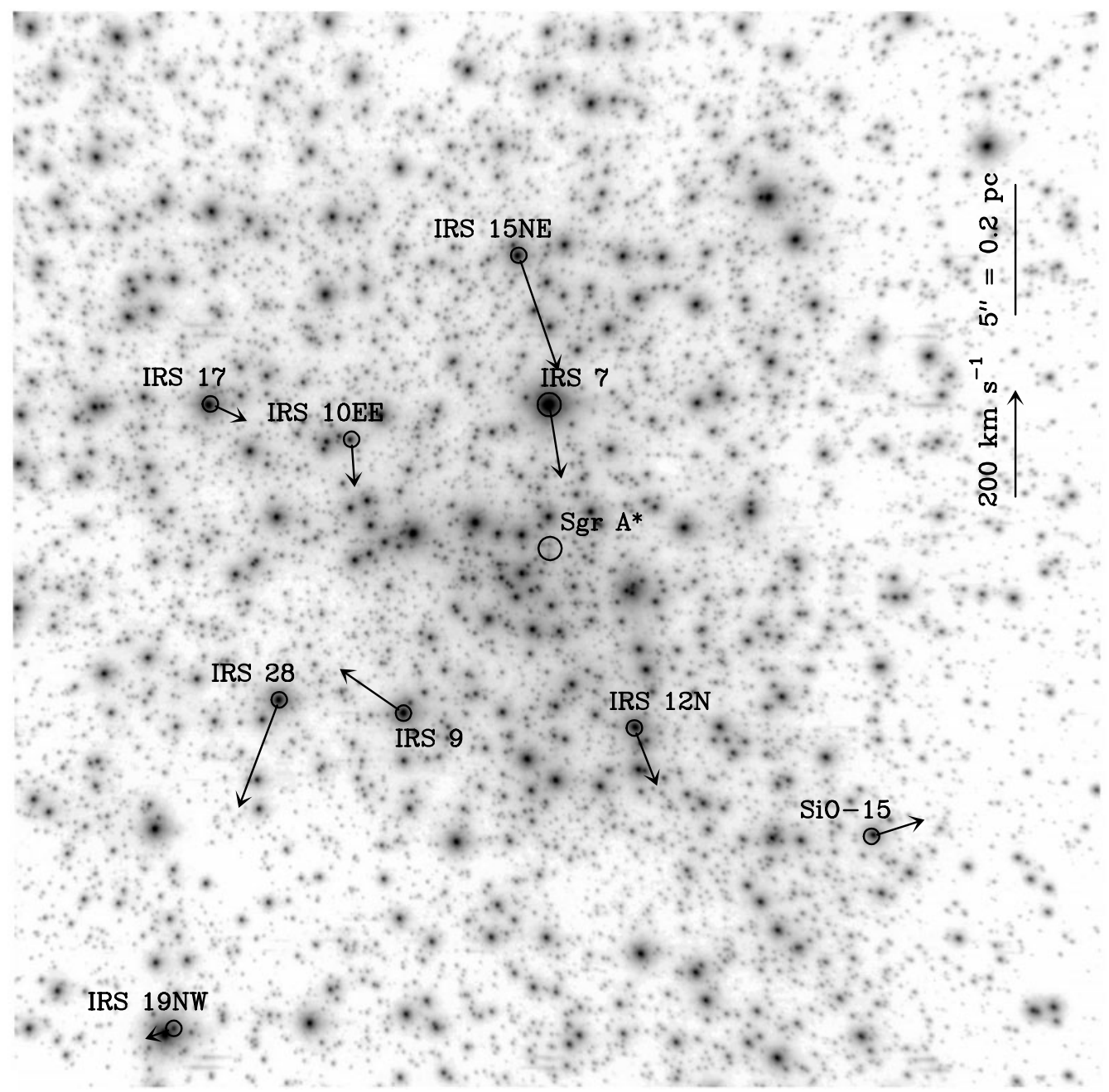

Figure 2. Infrared $\left(2.2 \mu \mathrm{m}\right.$ wavelength) image of the central $20^{\prime \prime}$ of the Galactic center from Reid et al. (2007). North is up and East is to the left. Stellar SiO masers are circled and their proper motions indicated by arrows. Linear and motion scales are at the right side of the figure. Sgr A*, a very weak infrared source, is circled at the center.

for a supermassive black hole at the Galactic center, one expects a very small motion of $\sim 0.3 \mathrm{~km} \mathrm{~s}^{-1}$ owing to small random perturbations from the $\sim 10^{6}$ stars (and perhaps upwards of $10^{4}$ stellar remnants) within Sgr $\mathrm{A}^{*}$ 's gravitational sphere of influence. With 8 years of VLBA data, we find that the true motion of Sgr $\mathrm{A}^{*}$ in the direction perpendicular to the Galactic plane is indeed very small: $-0.4 \pm 0.9 \mathrm{~km} \mathrm{~s}^{-1}$ (Reid \& Brunthaler (2004)).

The motion upper limit for Sgr A* can be directly transformed to a mass lower limit of $\sim 4 \times 10^{5} \mathrm{M}_{\odot}$. Combining this mass lower limit with an upper limit of $\approx 0.5 \mathrm{AU}$ for the intrinsic (non-scatter broadened) size of Sgr A* (see Bower et al. (2004) and references therein), yields a mass density $>7 \times 10^{21} \mathrm{M}_{\odot} \mathrm{pc}^{-3}$. This provides overwhelming evidence that $\operatorname{Sgr} \mathrm{A}^{*}$ is a supermassive black hole. 


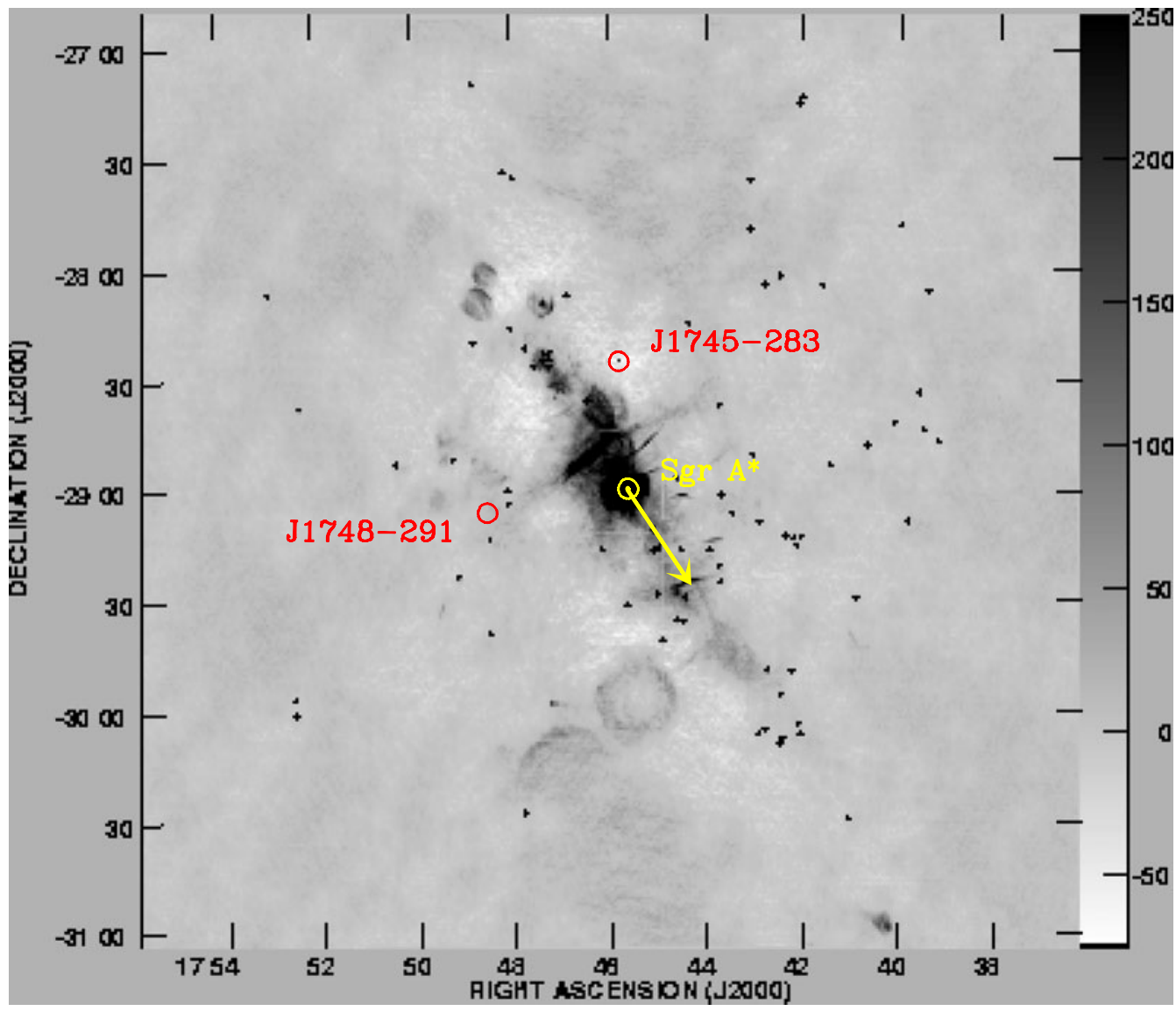

Figure 3. A schematic overlay of the location and expected direction of motion of $\mathrm{Sgr} \mathrm{A}^{*}$ on a 90-cm wavelength image of the Galactic center region (LaRosa et al. (2000)). Also shown are the locations of two compact extragalactic radio sources used as references for Sgr A*'s motion.

Since the apparent proper motion of $\mathrm{Sgr} \mathrm{A}^{*}$ is totally dominated by the orbit of the Sun about the Galactic center, one could use this to provide a dynamical definition of the plane of the Galaxy. The current IAU definition of the Galactic plane is based primarily on the distribution of HI, with the Sun defining the zero of Galactic longitude and latitude. The Sun is now known to be $\sim 10 \mathrm{pc}$ above the plane. The IAU definition of the Galactic plane, places Sgr $\mathrm{A}^{*} \approx 6 \mathrm{pc}$ below the plane. A better definition of the Galactic plane could come from the direction of the apparent proper motion of Sgr A* (corrected for the small peculiar motion of the Sun perpendicular to the plane), with Sgr A*'s position defining the zero of Galactic latitude. Of course, since the Sun orbits the Galactic center, the position of $\mathrm{Sgr} \mathrm{A}^{*}$ changes by about 6 mas $\mathrm{y}^{-1}$; thus the zero of Galactic longitude would become a function of time.

The accuracy of proper motions improves with the time span as $t^{-3 / 2}$ (for uniform time sampling). Our current data span 8 years; observations in 2007 will increase the time span to 11 years and should reduce our motion uncertainty by a factor of 0.6 . 

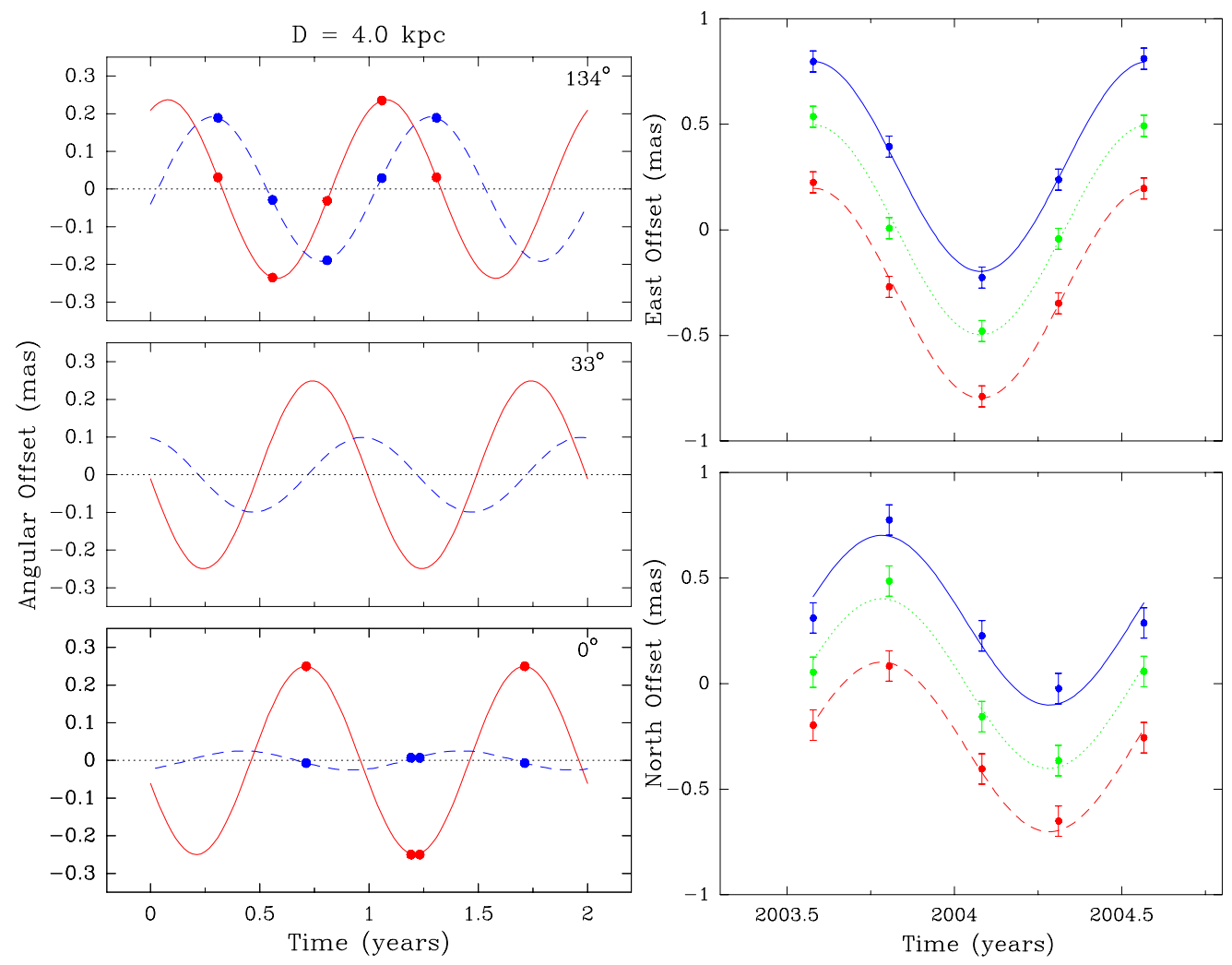

Figure 4. Left panels: Parallax signatures for three hypothetical sources at a distance of $4 \mathrm{kpc}$ at the Galactic longitudes indicated in the upper right-hand corners. Solid lines and dashed lines indicate the expected Eastward and Northward positions as a function of time after removing any proper motion. Near optimum time samplings are indicated for two cases. Right panels: Real parallax data from Xu, Reid, Zheng \& Menten (2006): VLBA positions of a $12.2 \mathrm{GHz}$ methanol maser spot of $\mathrm{W} 3 \mathrm{OH}$ (at Galactic longitude $134^{\circ}$ ) measured relative to three extragalactic sources (offset for clarity), after removing the fitted proper motion.

\section{Parallax and proper motions of star forming masers}

We are conducting a large program with the VLBA to map the spiral structure of the Milky Way and to define its rotational kinematics and mass profile. This involves measuring trigonometric parallaxes and proper motions to methanol (and some other) masers in massive star forming regions. We are observing primarily in the $12.2 \mathrm{GHz}$ methanol transition as that falls in one of the VLBA bands.

Examples of expected parallax signatures are shown in the left-hand panel of Fig. 4. Note that at Galactic longitude $134^{\circ}$ the Earth's orbit appears almost face-on as viewed by, eg, W3OH. For this case, one expects the amplitude of the Dec. parallax sinusoid to be nearly as large as the R.A. sinusoid. Sampling quarterly for one year, as shown, is a nearly optimum. However, at Galactic longitude $0^{\circ}$ the Earth's orbit appears almost edge-on as viewed by, eg, Sgr B2. For this case, the Dec. parallax sinusoid has a very low amplitude (and the VLBA beam is also bigger in this direction) and one can optimize the observations by measuring only the R.A. component at 6 month intervals as shown in the figure.

In the right-hand panels of Fig. 4, we show the $12.2 \mathrm{GHz}$ maser parallax data for W3OH by Xu, Reid, Zheng \& Menten (2006) with proper motions removed. These data 


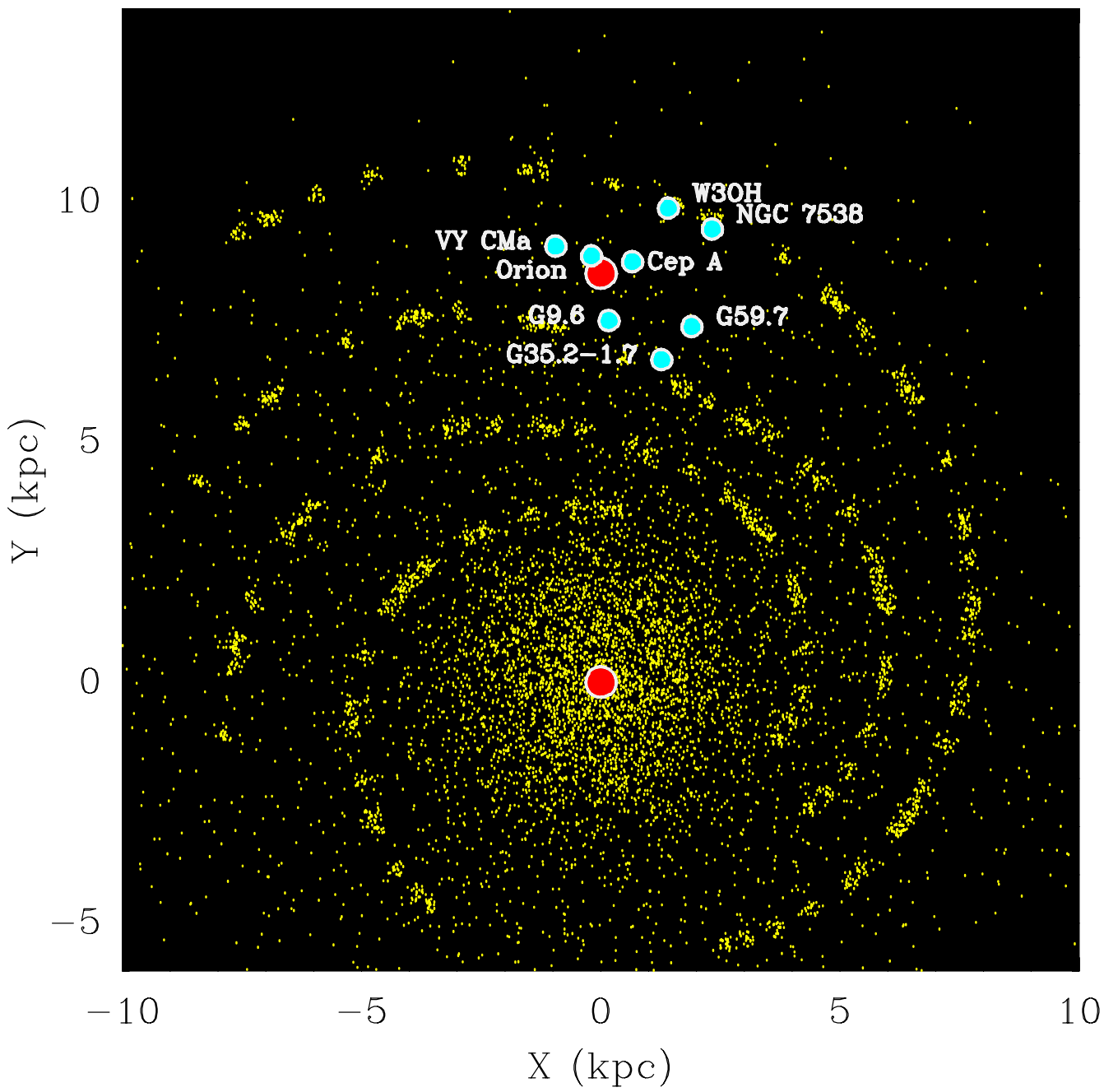

Figure 5. Schematic model of the Milky Way with spiral arms from Taylor \& Cordes (1993), with locations of massive star forming regions for which we have preliminary trigonometric parallaxes. The position of the Sun $(0,8)$ and Sgr A* $(0,0) \mathrm{kpc}$ are also indicated.

yield a parallax of $0.512 \pm 0.010$ mas (ie, a distance of $1.95 \pm 0.04 \mathrm{kpc}$ ). A similar parallax of $0.489 \pm 0.017$ mas, based on $\mathrm{H}_{2} \mathrm{O}$ masers in $\mathrm{W} 3 \mathrm{OH}$, has been measured by Hachisuka et al.(2006). This resolves a long-standing problem of the discrepancy of a factor of two between (associated O-star) luminosity-based distances of $2.2 \mathrm{kpc}$ by Humphreys (1978) and kinematic distances near $4.3 \mathrm{kpc}$ for this region of the Perseus spiral arm. The luminosity distance is consistent with our parallax and the kinematic distance is incorrect. We also measured the proper motion of $\mathrm{W} 3 \mathrm{OH}$, which reveals a large non-circular component of $22 \mathrm{~km} \mathrm{~s}^{-1}$ inward and slower than Galactic rotation (ie, roughly toward the Sun). This explains the large kinematic distance anomaly, but raises the question of how a massive star forming region could acquire such a large non-circular velocity component.

We are in the process of measuring parallaxes and proper motions for a dozen 12.2 $\mathrm{GHz}$ methanol maser sources, as well as some other star forming regions at other frequencies. While the observations are not complete, preliminary parallaxes for some sources are shown in Fig. 5. We have accurate distances to massive star forming regions in 


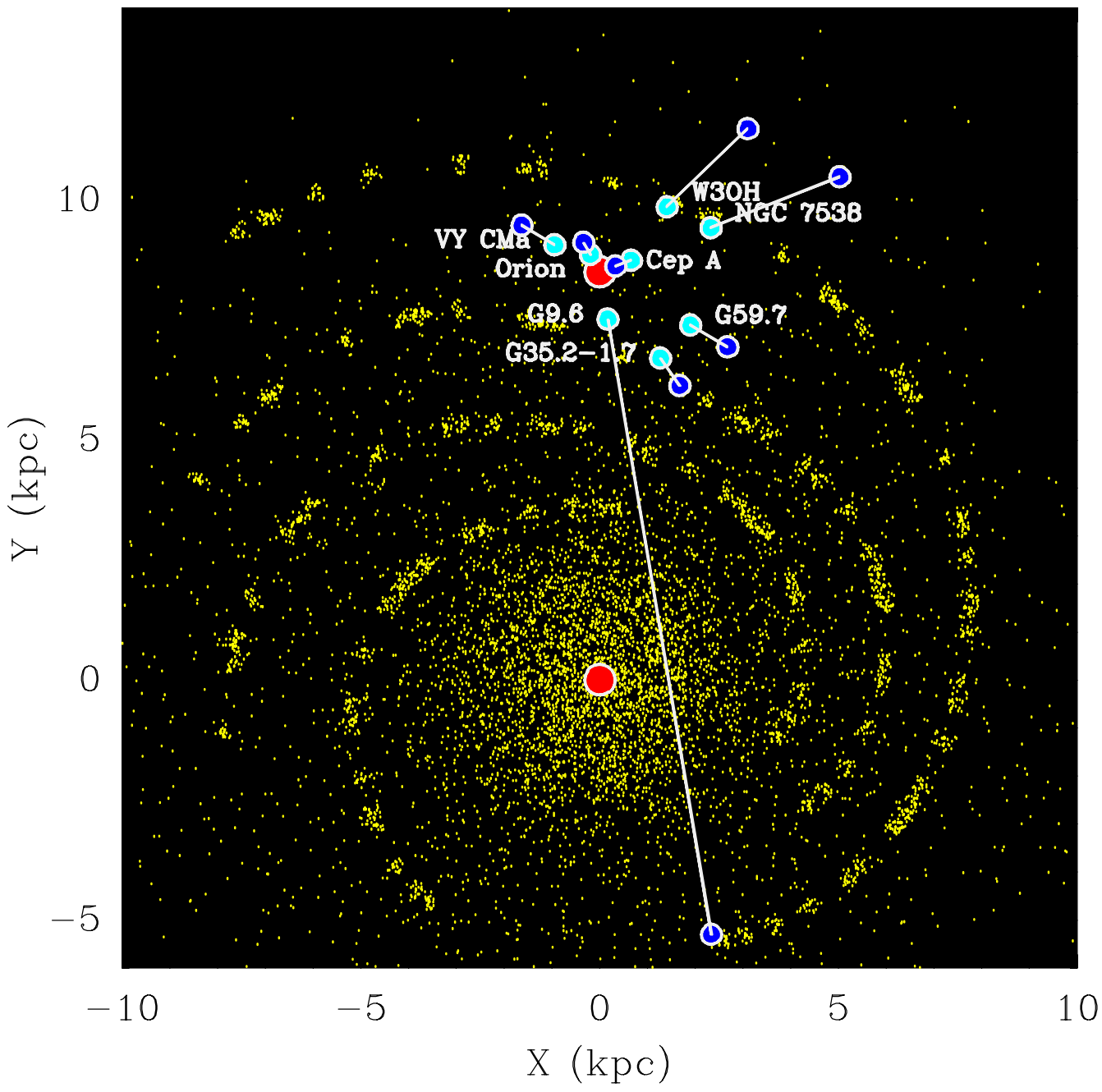

Figure 6. Comparison of trigonometric parallaxes (light cyan) with kinematic distances (dark blue), assuming $R_{0}=8.5 \mathrm{kpc}$ and a flat rotation curve with a local velocity of $\Theta_{0}=220 \mathrm{~km}$ $\mathrm{s}^{-1}$. For all sources, except Cep A, the kinematic distances are larger than the trigonometric parallaxes.

the Perseus, Local and Sagittarius arms. All sources, with the possible exception of G59.7+0.1, lie close to these spiral arms. With more measurements we plan to map the locations of the major spiral arms of the Galaxy.

In Fig. 6 we compare kinematic and trigonometric parallax distances. As for $\mathrm{W} 3 \mathrm{OH}$, we find the Perseus arm source NGC 7538 also has a large kinematic anomaly. The source G9.6+0.2 is generally thought to be at its far kinematic distance, well past the Galactic center. However, our preliminary (3-epoch) parallax indicates that it is nearby in the Sagittarius arm. This resolves the problem of the extreme luminosity of its $6.7 \mathrm{GHz}$ methanol maser, which was based on the far kinematic distance. Our measurements indicate that G9.6+0.2 is among the nearest high-mass star forming regions in the Galaxy.

Most sources appear to have trigonometric parallax distances that are nearer than their kinematic distances. Decreasing the galactocentric distance $R_{0}$ and/or increasing the rotational velocity of the solar neighborhood $\Theta_{0}$, from the adopted IAU 
values, would reduce the kinematic distances and bring them into better agreement with the trigonometric parallaxes. We note that the proper motion of Sgr A* by Reid \& Brunthaler (2004), after correcting for Solar Motion, implies $\Theta_{0} / R_{0}=29.45 \pm 0.15 \mathrm{~km}$ $\mathrm{s}^{-1} \mathrm{kpc}^{-1}$. If $R_{0}=8.0 \mathrm{kpc}$, this requires $\Theta_{0}=236 \mathrm{~km} \mathrm{~s}^{-1}$; using these Galactic parameters would reduce most kinematic distances by roughly $10-15 \%$.

We eagerly await more parallaxes from the VLBA and VERA.

\section{References}

Bower, G. C. Falcke, H. Herrnstein, R. M, Zhao, J.-H., Goss, W. M, \& Backer, D. C. 2004, Science 304, 704

Hachisuka, K. et al.2006, ApJ, 645, 337

Humphreys, R. M. 1978, ApJS 38, 309

LaRosa, T. N., Kassim, N. E., Lazio, T. Joseph W., \& Hyman, S. D. 2000, AJ, 119, 207

Menten, K. M., Reid, M. J, Eckart, A., \& Genzel R. 1997, ApJ 475, L111

Reid, M. J., Menten, K. M., Trippe, S, Ott, T., \& Genzel, R. 2007, ApJ, 659, 378

Reid, M. J., \& Brunthaler, A. 2004, ApJ 616, 872

Taylor, J. H., \& Cordes, J. M. 1993, ApJ 411, 674

Xu, Y., Reid, M. J., Zheng, X. W., \& Menten, K. M. 2006, Science, 311, 54 